\title{
A GOOD ALGORITHM FOR LEXICOGRAPHICALLY OPTIMAL FLOWS IN MULTI-TERMINAL NETWORKS
}

\author{
BY NIMROD MEGIDDO
}

Communicated by Alston S. Householder, December 27, 1976

\begin{abstract}
Let a network have several sources and sinks. For any flow $f$ let $\sigma^{f}$ and $\tau^{f}$ denote the vectors of net flows out of the sources and into the sinks, respectively, arranged in order of increasing magnitude. Our algorithm computes an $f$ for which both $\sigma^{f}$ and $\tau^{f}$ are lexicographic maxima. For a network with $n$ nodes this algorithm terminates within $O\left(n^{5}\right)$ operations.
\end{abstract}

1. The problem. A network $(N, c)$ consists of a set of nodes $N=\{1$, $\ldots, n\}(n \geqslant 1)$ and an $n \times n$ nonnegative matrix $c$ of capacities. $S \subset N$ is a nonempty set of sources and $T \subset N(T \cap S=\varnothing)$ is the set of sinks. A flow $f$ is an $n \times n$ matrix such that $0 \leqslant f_{i j} \leqslant c_{i j}(i, j \in N)$ and $\Sigma_{j=1}^{n}\left(f_{i j}-f_{i j}\right)=0$ for $i \notin S \cup T$. Denote $s=|S|, t=|T|$.

Let $\sigma^{f}\left[\tau^{f}\right]$ denote the $s$-tuple [t-tuple] of the numbers $\Sigma_{j=1}^{n}\left(f_{i j}-f_{j i}\right)$, $i \in S\left[\Sigma_{j=1}^{n}\left(f_{j i}-f_{i j}\right), i \in T\right]$ arranged in order of increasing magnitude. $f$ is called optimal if it maximizes both $\sigma^{f}$ and $\tau^{f}$ in the lexicographic orders on $R^{s}$ and $R^{t}$, respectively.

Optimal flows reduce to maximum flows (see [5]) when $s=t=1$. Existence of optimal flows is proved in [7]. The goal of this note is to present a good algorithm (in the sense of [2]) for finding an optimal flow.

2. The algorithm. The algorithm has two phases. In Phase I the network is decomposed to two networks, one with a single source and $t$ sinks, and the other with $s$ sources and a single sink. In Phase II optimal flows are found in these two networks.

Phase I. Find a flow $f$ which maximizes $\Sigma_{i \in S} \Sigma_{j=1}^{n}\left(f_{i j}-f_{j i}\right)$. Any of the following algorithms may be utilized: Karzanov [6] terminates within $O\left(n^{3}\right)$ operations, Dinic [1] and Even and Tarjan [4] $O\left(n^{4}\right)$, and Edmonds and Karp [3] $O\left(n^{5}\right)$. During the computation of $f$ a set $X, S \subset X \subset N \backslash T$, is generated such that for $i \in X$ and $j \notin X, f_{i j}=c_{i j}$ and $f_{j i}=0$. Next, construct the $X$. condensed and the $(N \backslash X)$-condensed networks (see [7]).

Phase II. Find optimal flows in the $X$-condensed and the $(N \backslash X)$-condensed networks independently. These two are treated symmetrically and, hence, without loss of generality assume that $S=\{1\}$.

AMS (MOS) subject classifications (1970). Primary 90B10, 90C35; Secondary 05C35, 94A20, 68A10.

Key words and phrases. Optimal flow, multi-terminal network, tree, polynomialtime algorithm. 
The following terminology is used throughout Phase II. The flow is the current flow through the network. An active sink is a sink $i$ such that it is possible to increase the net flow into $i$ without decreasing the net flows into other sinks. The graph is a directed graph whose set of nodes is $N$ and $(i, j)$ is an arc if and only if $f_{i j}-f_{i i}<c_{i j}$. The manual is the subgraph consisting of all nodes and arcs of the graph that lie on a shortest chain from the source to some active sink. The tree is a directed subtree of the manual satisfying: a. The source is the root of the tree. b. Every active sink is a node of the tree. c. Every tip of the tree is an active sink.

A rough description of Phase II follows. We start with the zero flow. The manual is constructed and a tree is chosen. Next, using tree-arcs only, we increase the net flows into active sinks equally until one of the tree-arcs leaves the graph. Then, if all the active sinks remain active, another tree is chosen and another maximum equal increase is employed as before; otherwise, the new manual is determined and again a tree is chosen. The algorithm terminates when the new manual is empty, or equivalently, when no sink is active. Specifically, Phase II is processed as follows.

Step 1. Construction of the manual. The set $M$ of the nodes of the manual is partitioned into layers $M_{0}, M_{1}, \ldots, M_{r}$. These are constructed as follows. First, set $M_{0}=S$. Then, set $M_{\nu}(\nu>0)$ to consist of all graph-nodes adjacent to nodes in $M_{\nu-1}$ but do not belong to $\bigcup_{\lambda=0}^{\nu-1} M_{\lambda}$. Let $M_{r}$ be the first layer such that either $\bigcup_{\lambda=0}^{r} M_{\lambda}$ contains all the active sinks, or $M_{r+1}=\varnothing$. In the latter case every sink $i \notin \bigcup_{\lambda=0}^{r} M_{\lambda}$ becomes inactive. If every sink is inactive, terminate. Next, every $i \in M_{r}$ which is not an active sink is deleted from $M_{r}$ and, recursively, every $i \in M_{\nu-1}$ which is neither an active sink nor adjacent to a node in $M_{\nu}$, is deleted from $M_{\nu-1}$. During the computation we maintain, for each $i \in M_{\nu}$, lists of the nodes in $M_{\nu-1}$ and $M_{\nu+1}$ which are adjacent to $i$.

Step 2. Construction of a tree. Every $i \in M_{r}$ is a node of the tree. Suppose that the part of the tree that connects the layers $M_{\nu}, \ldots, M_{r}(\nu \leqslant r)$ has been specified. For each $i \in M$ which either belongs to the tree or is an active sink, let $j$ be the first node in $M_{\nu-1}$ adjacent to $i$. Then $(j, i)$ also belongs to the tree. During the construction keep track of the numbers $v^{i}$ of active sinks $j$ such that $i$ lies on the unique chain of the tree that leads from the source to $j$.

Step 3. Flow change. Let $\epsilon$ be the minimum, taken over tree-arcs, of the numbers $\left(c_{i j}-f_{i j}+f_{j i}\right) / v^{j}$. For each tree-arc $(i, j)$, if $\epsilon v^{j} \leqslant f_{i i}$ set $f_{j i}=f_{i i}-$ $\epsilon v^{j}$; otherwise, set $f_{i j}=f_{i j}-f_{i i}+\epsilon v^{j}$ and $f_{j i}=0$.

Step 4. Manual change. During this step we maintain a list $L$ of manualarcs which are successively deleted. Initially, $L$ consists of those arcs of the last tree such that $f_{i j}-f_{i i}=c_{i j}$. Let $(i, j)$ be the first in $L$ which has not been deleted yet. If there is no $k \neq i$ such that $(k, j)$ is a manual-arc then add all manual-arcs of the form $(j, k)$ to the end of $L$. Similarly, all manual-arcs of the form $(k, i)$ are added to the end of $L$ if there is no manual-arc $(i, k)$ with $k \neq j$. 
Then $(i, j)$ is deleted and the next in $L$ is treated. Once $L=\varnothing$, if none of the active sinks has been deleted go to Step 2; otherwise, go to Step 1.

3. Discussion. Let $\nu^{i}$ be the index of that layer of the manual to which the active sink $i$ belongs currently $\left(0<\nu^{i}<n\right)$. Throughout the computation, $v^{i}$ does not decrease and at least one of the $v^{i}-s$ increases when a new manual is constructed. Thus, no more than $n^{2}$ manuals are constructed. A tree is constructed within $O(n)$ operations. A flow change results in a deletion of at least one manual-arc. A deletion of a manual-arc requires no more than $O(n)$ operations for updating $L$. Thus, since a manual has $n^{2}$ arcs at most, it will be deleted completely within $O\left(n^{3}\right)$ operations. This implies that the algorithm terminates within $O\left(n^{5}\right)$ operations. Optimality of the final flow follows from the standard theorem on max-flow and augmenting paths (see [5]).

\section{REFERENCES}

1. E. A. Dinic, Algorithm for solution of a problem of maximum flow in a network with power estimation, Dokl. Akad. Nauk SSSR 194 (1970), 754-757 = Soviet Math. Dokl. 11 (1970), 1277-1280. MR 44 \#5178.

2. J. Edmonds, Paths, trees, and flowers, Canad. J. Math. 17 (1965), 449-467. MR 31 \#2165.

3. J. Edmonds and R. M. Karp, Theoretical improvements in algorithm efficiency for network flow problems, J. Assoc. Comput. Mach. 19 (1972), 248-264.

4. S. Even and R. E. Tarjan, Network flow and testing graph connectivity, SIAM J. Comput. 4 (1975), 507-518.

5. L. R. Ford, Jr. and D. R. Fulkerson, Flows in networks, Princeton Univ. Press, Princeton, N. J., 1962. MR 28 \#2917.

6. A. V. Karzanov, Determining the maximal flow in a network by the method of preflows, Dokl. Akad. Nauk SSSR 215 (1974), 49-52 = Soviet Math. Dokl. 15 (1974), 434-437.

7. N. Megiddo, Optimal flows in networks with multiple sources and sinks, Math. Programming 7 (1974), 97-107. MR 50 \#15878.

DEPARTMENT OF STATISTICS, TEL AVIV UNIVERSITY, TEL AVIV, ISRAEL 